\title{
Cultura Cultura
}

Revista de Historia e Teria das ldeaias $\quad$ Revista de História e Teoria das Ideias

vol. 35 | 2016

Evidência, afecto e inconsciente

\section{À propos du refoulement}

affect, pulsion et inconscient selon la phénoménologie de Michel Henry

\section{Nuno Miguel Proença}

\section{(2) OpenEdition}

Journals

\section{Édition électronique}

URL : http://journals.openedition.org/cultura/2607

DOI : $10.4000 /$ cultura. 2607

ISSN : 2183-2021

Éditeur

Centro de História da Cultura

\section{Édition imprimée}

Date de publication : 1 décembre 2016

Pagination : 167-187

ISSN : 0870-4546

\section{Référence électronique}

Nuno Miguel Proença, « À propos du refoulement », Cultura [Online], vol. 35 | 2016, posto online no dia 05 fevereiro 2018, consultado a 06 maio 2019. URL : http://journals.openedition.org/cultura/2607 ; DOI : $10.4000 /$ cultura. 2607

Ce document a été généré automatiquement le 6 mai 2019.

(c) CHAM - Centro de Humanidades / Centre for the Humanities 


\title{
À propos du refoulement
}

\author{
affect, pulsion et inconscient selon la phénoménologie de Michel Henry
}

\author{
Nuno Miguel Proença
}

1 La lecture phénoménologique que Michel Henry fait de la psychanalyse est surprenante à plusieurs égards. En inscrivant Freud dans la lignée philosophique et en affirmant que ses thèses sur l'inconscient reprennent, sans le savoir, deux mouvements opposés de la métaphysique occidentale, Henry entend montrer que la psychanalyse doit passer outre le primat de la représentation pour être débarrassée des contradictions qui la traversent et qui la rendent prisonnière d'une philosophie de la conscience. Sans rejeter entièrement la théorie freudienne, l'auteur de la Généalogie de la Psychanalyse reformule le sens des notions de la métapsychologie en fonction de ses propres thèses sur le cogito, la subjectivité et l'auto-affection du vivant. Nous avons souhaité retracer les enjeux de cette redéfinition des pulsions, de l'affect et de l'inconscient à propos de ses réflexions sur le refoulement.

\section{Contexte des problèmes}

2 En situant quelques unes des notions clé de la théorie freudienne dans l'historial de la métaphysique occidentale, Michel Henry affirme, à la lumière de sa phénoménologie de la vie, la thèse selon laquelle le médecin de Vienne serait un héritier des élaborations métaphysiques qui l'on précédé - et ce malgré sa réserve affichée de celui-ci à l'égard de la philosophie. ${ }^{1}$ Au long de sa Généalogie de la Psychanalyse, l'auteur interroge ainsi le statut

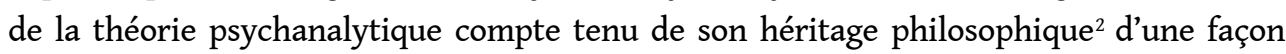
dont l'originalité n'est pas restée entièrement inaperçue dans le champ de la philosophie ${ }^{3}$ et ne devrait pas passer sans produire des effets dans celui de la psychanalyse. ${ }^{4}$ Mais, quelle influence les réflexions de Henry pourraient-elles avoir dans ce dernier? En quoi sa thèse sur la signification du concept d'inconscient ne devrait pas laisser indifférents ceux qui se consacrent au champ ouvert par Freud ? Pourquoi, au travers de cette recherche portant sur les précurseurs philosophiques qui ont rendu possible la découverte freudienne, les affirmations de Michel Henry « sont [-elles] en prise avec certaines des apories les plus fondamentales de la psychanalyse $»^{5}$ ? 
3 Dans un ensemble considérable de travaux, dont le plus important est sans doute la Généalogie de la Psychanalyse, mais dont il existe aussi des prolongements dans Phénoménologie Matérielle ${ }^{6}$ et dans plusieurs volumes de la Phénoménologie de la Vie, ${ }^{7}$ Michel Henry affirme tenter l'esquisse d'une nouvelle interprétation de la relation qui existe entre phénoménologie et psychanalyse, dans la mesure où il " propose une nouvelle conception de la phénoménologie ${ }^{8}$ Bien que la philosophie de Michel Henry s'inscrive dans le courant phénoménologique, elle le dépasse, en effet, par l'élaboration du concept d'être du sujet en sa phénoménalité immanente et originaire et par sa tentative de le décrire. Si, chez Husserl, la conscience intentionnelle est le principe constituant des phénomènes et la condition de tout savoir, Henry dira du sujet et de son auto-réceptivité qu'il est le seul fondement authentique de la connaissance et de l'expérience. Il critiquera alors l'assimilation de l'essence à l'idée et la réduction de l'être à sa seule manifestation objective, c'est-à-dire à la représentation. Par ailleurs, il critiquera aussi l'ontologie heideggérienne et son affirmation de la transcendance de l'être.

4 Extériorité, visibilité et transcendance, en tant que structures du champ idéel du savoir objectif, reposent sur un pouvoir plus originaire, sur un principe substantiel inhérent à la subjectivité. L'ego transcendantal est ouvert au monde par un pouvoir qui lui est imanente et qui en assure également la réceptivité ek-statique. L'objectivité requiert l'auto-réceptivité du sujet et s'y fonde. Ce pouvoir, qui assure la dynamique d'ouverture à l'extérieur, s'accomplit dans l'épreuve concrète de soi d'une subjectivité, dans l'autoaffection d'une vie en tant que jouissance et souffrance. Le travail de Henry consistera, alors, dans l'élaboration d'une ontologie de la subjectivité (même si le terme n'est pas le plus correct, précisément parce que la Vie n'est pas, ${ }^{9}$ si nous entendons l'être de manière ek-statique). Une telle vie, indivisiblement corps et esprit, définit l'essence de la réalité humaine en tant qu'affective et sensible, de même qu'elle détermine les modalités théoriques et pratiques de l'existence qui lui sont subordonnées. Le domaine des sciences - d'une part -, celui de la culture, de l'économie et de la politique - d'autre part - sont subordonnés à la Vie qui se trouve à l'origine de toute activité et de toute réalisation humaines. Toutefois, alors qu'elle décèle une hiérarchie entre le principe dont ils sont issus et les différents domaines de l'activité humaine (ontologiquement secondaires par rapport à celui-là), la pensée henryenne rend également compte d'une inversion axiologique entre l'ordre de l'idéalité - dont relèvent les catégories de la science et les entités sociales et politiques - et celui dont procèdent les modalités sensibles et affectives de la vie subjective, dont relèvent l'art et la culture. Cette inversion aurait pour conséquence d'innombrables crises et catástrofes de notre temps. En valorisant les premiers au détriment des seconds, c'est l'objectivité qui dénie la subjectivité dont elle est issue et les représentations dont est fait le monde qui cachent l'activité qui en est à l'origine.

5 Contrairement aux sciences humaines, la psychanalyse « résiste à la réduction galiléenne ${ }^{10}$ par cela qu'elle « affirme et maintient, fût-ce sans le savoir, le droit invincible de la vie ${ }^{11}$ dans ce que celle-ci a d'irrémédiablement subjectif, alors que l'humanité est ravagée par le savoir objectiviste et par ses prétentions exorbitantes. Cela ne l'écarte pourtant pas entièrement de l'effort d'objectivation de la vie subjective. Si la psychanalyse reconnaît, en effet, " l'immanence radicale de la subjectivité absolue dans son hétérogénéité ontologique au monde de la représentation $",{ }^{12}$ elle maintient, toutefois, le présupposé scientiste selon lequel seul existe ce qui est objectif et déterminable objectivement : " l'inconscient psychique, reconnu finalement comme l'affect, n'est encore que le 
représentant de processus bioénergétiques, donc d'une réalité naturelle ${ }^{13} \cdot{ }^{13}$ Cette double tendance serait, en outre, renforcée par la signification double de la notion d'inconscient, donnant de la sorte origine à une tension au sein de la théorie psychanalytique et se traduisant, selon Henry, par un mouvement contradictoire au niveau de la clinique et des formes de connaissance (de soi) qu'elle rend possibles.

Henry repère ainsi chez Freud à la fois les restes de la métaphysique occidentale centrée sur le cogito - dont le médecin de Vienne serait, à son insu, un " épigone $»^{14}$ et un " héritier tardif $»^{15}$ - mais aussi un dépassement de cette même métaphysique, rendu possible par la Volonté schopenhauerienne et par la Vie nietzschéenne. Selon Henry, la compréhension psychanalytique de la psyché serait en partie une conséquence de la réduction de l'essence originelle de la phénoménalité à la représentation, telle qu'elle aurait été opérée par une certaine lecture du cogito cartésien, tout en étant une continuation de ce que la réflexion débutée par l'auteur de Le Monde comme volonté et comme représentation et différemment prolongée par Nietzsche nous donnent à entendre d'une Vie irréductible à la phénoménalité dont elle est formatrice. La psychanalyse redécouvre ainsi, selon Henry, l'importance décisive de la vie et de son pathos, dont l'essence est pulsionnelle. C'est à ce dernier titre que la Généalogie de la Psychanalyse reconnaît à la théorie freudienne " une portée ontologique immense ${ }^{16}$ par cela qu'elle affirme, en effet, que le «fond de la psyché $~^{17}$ échappe à jamais à la pensée et à son Ekstase représentative, qu'il se situe en deçà de celle-ci et la conditionne. À ce fond irreprésentable, dont la force est à l'origine de la représentation et de l'affect qui l'accompagne, la psychanalyse donne le nom d'inconscient. En tant que telle, la découverte freudienne serait alors le dernier terme de la reconnaissance d'une subjectivité où se trouve ce même savoir, à une époque où prime une idéologie de l'objectivité et de l'extériorité des sources de la connaissance. Mais, d'autre part, en tant qu'elle est traversée par une visée scientifique, et alors même qu'elle découvre la source du savoir là où l'activité subjective a sa source, la psychanalyse souhaiterait se débarrasser de celle-ci et agirait à sa façon le désespoir qui consiste à vouloir (s')arracher une partie de soi-même en séparant l'activité propre à la pensée représentative (où se situe la clinique, dès lors qu'elle met l'accent sur le retour à la conscience et sur l'aspect verbal et interprétatif du travail thérapeutique) de la passibilité subjective. Le travail de la cure aurait, alors, en partie pour tâche de placer la seconde sous les logiques de la première en reconduisant au plus proche de zéro l'excitation à l'origine de toute l'activité pulsionnelle non inféodée à l'ordre de la signification consciente et verbalisable. Autrement dit, malgré son hypothèse de l'inconscient, la psychanalyse ne serait pas capable de sortir de l'ordre des représentations et elle finirait - en dépit de ses hypothèses métapsychologiques - par ne prendre en considération qu'un inconscient qui n'est que le revers nécessaire de la conscience et qui n'est fait que de ses contenus latents.

7 L'intérêt de la lecture henryenne ne concerne pas seulement la réflexion philosophique sur la psychanalyse, il concerne aussi, plus spécifiquement, le paradoxe apparent d'une approche phénoménologique de l'inconscient et des notions d'affect et de pulsion. En quoi la critique de la signification double de l'inconscient (un inconscient de la " représentéité » et un inconscient «efficient»), telle qu'elle est proposée par la phénoménologie radicale de Michel Henry, serait-elle en mesure de fournir les éléments pour dépasser la contradiction qui advient de la rencontre entre une méthode centrée sur la description des vécus et des configurations immanentes de la conscience et une théorie qui place le centre de la vie mentale ailleurs que dans la conscience ? Parmi les difficultés 
de cette rencontre, nous trouvons celle qui est soulevée par la thèse freudienne concernant le refoulement. Comment la caractérisation henryenne d'une subjectivité absolue, dont l'essence est l'auto-affection de la vie, intègre-t-elle ce phénomène décrit par Freud? Autrement dit, que signifie le refoulement pour une phénoménologie qui affirme que « l'inconscient n'existe pas » ${ }^{18}$ ?

\section{Que veut dire « refoulement »?}

8 Dans la métapsychologie freudienne, rappelons-le, le concept d'inconscient est indissociable de celui de refoulement. C'est à cause de ce à quoi Freud donne le nom de "refoulement" que le centre de la psyché est déplacé et qu'il n'est plus identifiable à la conscience et à ses représentations. C'est en raison du refoulement que l'inconscient est formé comme zone inaccessible à celles-là, régie par un processus singulier (le processos primaire) à l'oeuvre de façon manifeste dans le travail du rêve, dans le lapsus, dans le mot d'esprit et dans la formation de symptômes. C'est dans cette même zone maintenue à l'écart de la conscience par un effort constant (le refoulement) que se trouve, selon Freud, le centre de la vie psychique. La lecture du concept d'inconscient, telle qu'elle est faite par Michel Henry dans sa Généalogie de la Psychanalyse, semble radicaliser ce déplacement. Mais, cette radicalisation de l'effort pour situer ce qu'il y a d'essentiel à la vie ailleurs que dans la conscience représentative conduit l'auteur de la Généalogie de la Psychanalyse à une transformation des concepts d'affect et de pulsion tels qu'ils sont requis pour rendre compte du refoulement. Si Henry s'appuie sur l'hypothèse freudienne selon laquelle c'est parce qu'on ne peut se fuir soi-même que le refoulement a lieu, l'originalité de sa lecture attire notre attention sur le fait que la force inhérente au refoulement est d'ordre affectif, qu'elle n'est pas un effort pour maintenir les représentations à distance, mais qu'elle est plutôt formatrice des représentations par le biais de ses propres teneurs et de leur force. L'énergie à l'oeuvre dans le refoulement est celle de la vie elle-même, par rapport à laquelle il n'y a pas d'écart possible : l'inconscient, plutôt qu'une zone de représentations séparée de la conscience, se confondrait avec l'activité incessante de la vie, en tant qu'elle s'éprouve dans la dynamique affective, au point de ne pas exister.

9 Quels sont, donc, les arguments sur lesquels se fonde cette lecture qui relie de façon originale inconscient et refoulement et qui semble mener, pour reprendre les termes de Mikel Borch-Jakobsen, à «une affirmation aussi énorme, aussi insensée» ${ }^{19}$ en apparence, selon laquelle "l'inconscient, à la fin, n'est rien d'autre que [la] conscience même "? ${ }^{20}$ Quel rapport y a-t-il entre, d'une part, le décentrement de la conscience, tel qu'il est affirmé par Freud à propos de l'effort pour tenir à l'écart les représentations et, d'autre part, l'auto-affection de la vie et l'ipséité pathique en tant qu'ils sont identifiés par Henry comme des composantes essentielles de la subjectivité?

Selon l'hypothèse freudienne, le décentrement de la conscience, son conditionnement par des processus qui ne relèvent pas de son initiative, est à l'origine de la constitution d'une zone psychique inconsciente à la seule condition que les représentants de la vie organique au sein de la vie psychique fassent l'objet d'un refoulement. Par le biais de cette notion, Freud rend compte d'un jeu de forces en raison duquel est constituée une zone de la vie psychique composée par cela qui, non seulement n'est pas en mesure d'accéder à la conscience, mais ne cesse - pour autant - d'avoir des effets sur le cours de la vie consciente et de ses représentations. La formation, au sein de la vie psychique, d'une zone inconsciente destitue le moi du rôle central qu'il s'y arrogeait et inflige à 
l'humanité, selon Freud, la dernière d'une série de blessures narcissiques qui doivent l'inviter à l'humilité : le Moi n'est pas maître dans sa propre maison. C'est l'inconscient qui y règne. Mais de quel inconscient s'agit-il ? D'un ensemble de représentations, d'un ensemble de processus? Ou bien s'agit-il encore d'autre chose?

11 Outre les processus psychiques qui ne deviennent accessibles et subordonnés au moi que par une perception incomplète et incertaine, notre vie révèle une passivité de fond en raison de laquelle, en nous apparaissant, nous nous apparaissons toujours impliques dans un déjà-là d'une vie dont nous ne sommes pas à l'origine et qui nous situe dans un méandre de sensations et de besoins, de significations et de désirs, entrelacées à des interdits, à des règles et à des usages qui ne relèvent pas de notre initiative et qui conditionnent notre vie vigile et intentionnelle. Passivité à l'égard des constellations de sens véhiculés par des représentations et passivité à l'égard du sens véhiculé par les dispositions éprouvées. Une vie est chaque fois passible des transformations issues de et provoquées par ce double registre de la passivité. Dans le vocabulaire de Henry, le premier est ek-statique, le deuxième est immanent. Ce ne sont pas seulement la faim, la soif, les besoins qui résultent de l'ingestion et de la digestion, la respiration et autres efforts d'autoconservation issus d'une activité incessante qui entrelacent la vie consciente au dynamisme d'un corps vivant. Ce sont aussi le plaisir et le désir sexués. Or, selon Freud, ce qui fait le plus souvent l'objet d'un refoulement donnant lieu à la formation d'une zone psychique inconsciente qui déplace le centre de la vie psychique vers une zone autre que celle du Moi ce sont précisément les pensées, les images ou les souvenirs liés aux pulsions sexuelles. Mais pourquoi y a-t-il refoulement, est-ce en raison des dynamiques immanentes ou bien en raison d'une logique des représentations?

Le refoulement, en tant qu'il est « la pierre angulaire de l'édifice de la psychanalyse ${ }^{21}$ est un des quatre destins possibles des pulsions. Dans le lexique freudien, la Trieb apparaît définie par les notions de " source ", " but ", « objet » et " poussée ».22 Elle est un processos dynamique, qui consiste dans une charge énergétique, elle-même facteur de motricité : c'est cette charge que Freud nomme "poussée » et en raison de laquelle l'organisme tend vers un « but ». La « source » de la pulsion est une excitation corporelle, qui est l'état de tension faisant pression et son « but » est de supprimer la tension qui se situe à sa source. La pulsion atteint son but grâce à son " objet ». C'est en lui, ou par lui, que la tension issue de la source, et créant une pression, est supprimée : c'est là ce que l'on identifie comme étant le plaisir, ou la satisfaction, et qui constitue le véritable but de la pulsion. Cela a toute son importance pour la lecture de Henry. Mais, de façon à souligner l'emploi que celui-ci va faire des conclusions freudiennes pour sa propre ontologie, ${ }^{23}$ remémorons la différence établie par l'auteur de la Métapsychologie entre les excitations internes et les excitations externes. Cela est essentiel pour comprendre la signification que l'affectivité peut avoir pour une phénoménologie après que les notions de plaisir et de déplaisir vont être transformées par la deuxième théorie des pulsions.

13 À la différence des excitations qui viennent de l'extérieur, qui ont un impact unique et qui peuvent être supprimées par l'emploi de leur charge énergétique dans un type d'action approprié, comme la fuite, les excitations pulsionnelles ont une force d'impact permanent, qui est indice du monde intérieur. Si les excitations, dont la physiologie a donné le concept, sont rattachées au schéma du réflexe - selon lequel l'excitation qui est apportée de l'extérieur au tissu vivant est également déchargée vers l'extérieur sous la forme d'une action unique appropriée, qui soustrait la substance excitée à l'effet de l'excitation et l'éloigne de son champ d'influence - l'excitation pulsionnelle, en revanche, en venant de 
l'intérieur de l'organisme, agit de manière différente sur le psychique et exige d'autres actions qui seront seules capables de les éliminer. Elle " attaque de l'intérieur $»^{24} \mathrm{du}$ corps. Sa force d'impact est " constante $",{ }^{25}$ et jamais " momentanée $»{ }^{26}$ Ses destins essentiels, puisqu'on ne peut en supprimer la source (ce qui reviendrait à se supprimer soi-même), sont donc quatre : l'inversion dans leur contraire, le directionnement sur la personne propre, le refoulement ou la sublimation. Mais en aucun cas la fuite. Selon Freud, le troisième de ces destins, le refoulement, est une négation spécifique des pulsions, qui ne les détruit pas, mais qui les suspend. Son essence consiste ainsi à " écarter et maintenir à distance du conscient $\aleph^{27}$ les représentants des pulsions et à les circonscrire dans cette zone de la vie psychique nommée inconscient. Sont ainsi refoulés les représentants des pulsions dont la satisfaction provoquerait du déplaisir, ou plutôt (et parce que Freud pose que le but de la pulsion est la satisfaction), sont refoulées celles dont la satisfaction provoquerait simultanément plaisir et déplaisir, ce dernier étant en quantité supérieure. Quelle que soit son intensité, néanmoins, la force d'inhibition provoquée par le refoulement ne peut entièrement détruire ni la pression pulsionnelle, ni sa source (ce qui équivaudrait à supprimer l'organisme vivant). Son type de refus particulier ne détruit pas non plus ses représentants demeurés inconscients sous forme de représentations. Mais qu'en est-il de l'affect par lequel se traduit la pulsion, connaît-il le même sort que la représentation ? Si, parfois on parle d'affects refoulés, "le refoulement ne signifie donc ici aucune disparition de l'affect ni par conséquent de la phénoménalité qui lui appartient par principe, mais seulement sa modalisation en un autre affect $»{ }^{28} \mathrm{Et}$, tandis que, dans le refoulement, la représentation liée à l'affect n'est pas formée, «l'affect lui-même n'est pas supprimé mais modifié qualitativement, devenant telle ou telle tonalité. Quand celles-ci à leur tour sont interdites, refoulées, c'est dans l'angoisse qu'elles se changent en même temps que l'affect primitif $» .{ }^{29}$ Cette affectivité indépassable (en deçà de laquelle il n'y a rien que l'activité incessante du corps vivant et l'ensemble de ses pouvoirs et de ses forces) révèlerait une subjectivité incarnée qui trouve son activité enracinée dans une passibilité de fond, celle de la Vie, avant le monde et ses représentations. Henry signale ici la passibilité foncière de l'ipséité : autrement dit, " la constance de l'affection et le fait qu'il ne soit pas possible de lui échapper pour la fuir, de développer un écart, une différence, de prendre le moindre recul à son égard, c'est-à-dire à l'égard de soi-même ne qualifie rien d'autre que la subjectivité absolue et ainsi, en tant qu'affection immanente de soi par soi, l'essence de l'ipséité, et par conséquent, de cette façon, le moi (non plus nommé de l'extérieur, mais impliqué dans sa passibilité la plus intérieure et la plus inaliénable) $» .^{30}$

14 Prise en soi-même et prisonnière de soi-même, une telle affection - bien qu'étant le résultat d'une poussée transformatrice qui lui est inhérente - est auto-affection et la subjectivité absolue se confond avec l'activité incessante, dont l'initiative ne lui revient pas, ce que Freud nomme " source d'excitation " à l'origine des pulsions. La force interne et formatrice de la vie se révèle ainsi au vivant, dont le corps est le lieu. C'est là que l'autorévélation affective de la vie a lieu en tant qu'elle est vécue par un vivant toujours déjà implique dans une communauté dont l'essence est la vie. Cela veut-il dire, pour autant, que la subjectivité n'est plus travaillée par une altérité et que Michel Henry efface la différence entre conscient et inconscient et? ${ }^{31}$ Si la phénoménologie henryenne insiste sur l'identité entre la vie et ce que Freud nomme l'excitation endogène en en faisant l'essence de l'ipséité, elle souligne aussi le fait que personne n'est à l'origine de cette vie dont le dynamisme affectif se révèle en tant que Soi. Ainsi Henry reprend-il "de l'intérieur" la description freudienne de l'excitation pulsionnelle pour en faire un 
élément essentiel de la vie tout en le liant à l'affectivité et en en faisant sa force inhérente. "Cet effort de la vie pour modifier sa tonalité et pour se modifier soi-même c'est la pulsion. Et voilà comment la vie dans son affectivité et en raison de celle-ci devient cette tension intérieure inlassable elle aussi par laquelle elle s'efforce sans cesse de se transformer soi-même, très précisément de changer le malaise qui lui vient de sa propre subjectivité en autre chose de plus supportable - comment l'Affect se fait Force ». 32

\section{Quel rapport y a-t-il entre Schopenhauer et Freud à propos du refoulement?}

Le rapport particulier entre la Force, la Forme et l'affectivité, décelé ici par le biais d'une réflexion sur l'essence du refoulement et retrouvé dans les descriptions phénoménologiques de la pulsion, dont nous ne donnons qu'un exemple, permet à Henry d'identifier un des héritages métaphysiques de Freud. Il s'agit d'une ontologie nouvelle, celle de Schopenhauer qui, d'après l'auteur de la Généalogie de la Psychanalyse, " découvre l'affectivité comme révélation de l'Etre en lui-même, comme la matière dont il est fait, comme sa substance et sa chair $\gg \cdot{ }^{33}$ Freud s'inscrit, d'autant plus dans cette lignée de métaphysiciens pour lesquels la manifestation phénoménale ne saurait se réduire à la représentation que le concept de refoulement lui-même a été introduit pour la première fois dans la pensée occidentale par Schopenhauer comme " processus psychique par lequel certains faits euxmêmes psychiques en repoussent d'autres, leur interdisant, sinon l'existence, du moins l'accès à la conscience ${ }^{34}{ }^{34}$ Cette inscription est elle aussi d'autant plus claire qu'au dire de Freud lui-même, nous rappelle Henry, la notion schopenhauerienne de volonté " équivaut aux pulsions de la psychanalyse ${ }^{35}$ et le rapport qu'il existe entre volonté et représentation chez Schopenhauer est analogue à celui qui existe entre pulsions et représentations chez Freud. Cette Volonté qui ne peut pas ne pas vouloir est semblable aux désirs qui laminent notre corps, ou plutôt identique à eux, elle est cette activité toujours recommencée, qui nous traverse, que nous sommes et à laquelle il ne nous est pas possible d'échapper. Le rapport qu'elle entretient avec l'affectivité et leur situation respective à l'égard de l'essence de la vie se décide dans " l'analyse extraordinaire où se trouve reconnu pour la première fois et cerné dans sa possibilité le phénomène du refoulement $»{ }^{36}$ Henry trouve ainsi le concept de refoulement à propos de trois problèmes auxquels Schopenhauer allait " apporter des solutions absolument originales " : celui de la mémoire, celui de la perception et celui du rapport entre raison et folie $»{ }^{37}$ Demandons-nous, en les reprenant, comment, par le biais de la présentation qu'il en fait, Henry y trouve les arguments à la fois pour inscrire Freud dans la lignée de Schopenhauer et, ce faisant, pour déplacer la problématique du refoulement du champ dessiné par l'opposition entre le manifeste et le latent - qui est dépendant de celui de la représentation - vers celui de l'affectivité, qui n'est dite inconsciente que de façon incorrecte,$^{38}$ de façon à interroger la signification de l'inconscient.

La théorie schopenhauerienne de la mémoire est formulée à partir de la caractérisation générale du rapport entre volonté et représentation. Entendue selon la doctrine de Schopenhauer, une représentation est un double irréel de la force que constitue la volonté. Si la première est entièrement " passive », ${ }^{39}$ la seconde est « toute-puissante $»^{40}$;

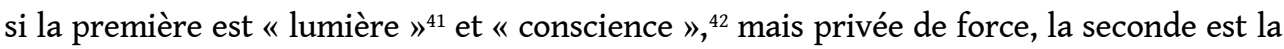
force, mais elle est " privée de la représentation et de sa lumière ", ${ }^{43}$ de telle façon que ces 
deux instances se définissent par leur " exclusion réciproque $»{ }^{44}$ C'est parce que, dans son analyse, « la volonté est sans représentation et la représentation sans volonté $»^{45}$ que la théorie schopenhauerienne du psychisme peut être dite " une théorie du refoulement " ${ }^{46}$ qui a déjà en commun avec la théorie de Freud le fait que la vie de la conscience est déterminée par cela qui n'a pas de lumière, c'est-à-dire, " par le pouvoir lui-même aveugle de nos instincts et de nos pulsions $»{ }^{47} \mathrm{Si}$ l'essence de celui-ci est définie comme ne consistant que dans le fait d'écarter et de maintenir à distance du conscient, nous la retrouvons chez Schopenhauer lorsqu'il nous signale que, lors du rappel du souvenir ou de son rejet, la formation de la représentation s'explique par la Volonté. «Telle ou telle représentation particulière est ou n'est pas voulue et, dans ce dernier cas, c'est la volonté qui "la tient en quelque sorte couverte de la main" ". ${ }^{48}$

La représentation ainsi recouverte demeure bien, mais elle n'est pas admise dans la conscience. Son rejet équivaut à la forme particulière de négation du refoulement telle que Freud la définit, qui ne détruit pas mais conserve, et qui s'accompagne d'un savoir. Henry nous rappelle que ce processus est exactement celui que Freud a décrit dès 1893 dans les Études sur l'hystérie : il s'agissait de choses que le malade voulait oublier et qu'intentionnellement il maintenait, repoussait hors de sa pensée consciente. La mémoire, chez Schopenhauer, mais aussi chez Freud, n'est une faculté de la représentation qu'en apparence. Le contenu représenté est conduit devant l'esprit, non pas par une capacite de le pro-duire en tant qu'objet, mais par " le pouvoir, en soi étranger, à cette production, qui lui permet de s'accomplir ou qui le lui interdit $\gg .{ }^{49}$ Cela se traduit, chez Schopenhauer comme chez Freud, par une rupture du fil de la mémoire. L'absence qui vient rompre et briser le fil de la mémoire et la chaîne des représentations est un pouvoir autre, qui interdit la représentation. Il s'agit de la volonté, en tant qu'elle est à la fois la condition de la mémoire et de l'oubli : " ne pas vouloir que tel contenu entre dans la lumière de la représentation relève du même principe que celui qui soutient la mémoire, en est la simple détermination négative $» .^{50}$

18 C'est donc bien le refoulement qui se trouve ainsi défini par Schopenhauer, avec cette exclusion par la volonté d'une représentation hors du domaine de la conscience. Dans cet établissement du lien généalogique qui existe entre le concept de refoulement tel qu'il se dessine chez Schopenhauer et de celui qui est développé par Freud, nous apprenons également que la dichotomie entre le vouloir-vivre et les représentations est constitutive de la Psyché. Le fait que l'instinct aveugle détermine la conscience représentative est « la loi générale ${ }^{51}$ du psychisme. Il s'agit, nous dit Henry, de "l'expression permanente et incontournable de sa structure essentielle ${ }^{52}$ de celle-là qui, en termes freudiens, conduira à une subordination du Moi aux forces inconscientes en raison desquelles il ne sera plus maitre de sa maison. Autrement dit, le décentrement provoqué par le refoulement se trouve déjà chez Schopenhauer, de même que la « manipulation de la représentation par la pulsion $»^{53}$ attestée par toute vie imaginaire et en raison de laquelle l'association d'idées est utilisée par la psychanalyse pour tenter de dévoiler l'inconscient. C'est parce que le rapport de condition à conditionné qui existe entre le Vouloir et la représentation se retrouve chez Freud comme rapport entre inconscient et conscient que le travail clinique se proposera comme tâche d'induire le second à partir du premier précisément en levant les refoulements. Or, se demande Henry, pourquoi la volonté (comme le désir ou la pulsion) refoule-t-elle la représentation? « Non pas à cause de son contenu représentatif mais de son affectivité $~^{54}$ et, " si le refoulement appartient à la constitution fondamentale de la psyché comme une de ses lois les plus constantes et les 
plus profondes, c'est d'abord parce que la représentation qu'il s'agit de refouler - ou d'accueillir - est déterminée affectivement [;] c'est en raison de cette affectivité seulement qu'elle est l'objet d'un rejet et que celui-ci peut et doit se produire $» .^{55}$

Il en va de la perception comme de la mémoire et de l'oubli : c'est encore la Volonté qui voudra ou ne voudra pas. Certains fragments du champ perceptif seront ou ne seront pas voulus. L'ouverture et l'anticipation perceptives seront façonnées par la force formelle de la volonté en tant que celle-ci portera ou détournera le regard en « rejetant alors un objet ou une partie de cet objet hors représentation et ainsi hors conscience, et le maintenant, à distance du conscient, comme le dit Freud $\|^{56}$ en raison de ce qu'il suscite. Autrement dit, c'est parce qu'une représentation est affectivement déterminée ou investie d'affect qu'il peut y avoir un refus de son entrée dans l'ensemble de celles qui composent la vie consciente, ce n'est pas à cause de son contenu. Michel Henry souligne, à ce propos, les expressions de Schopenhauer qui font référence au chagrin causé par une pensée, à la cruauté d'un souvenir qui devient pour cela insupportable, à l'angoisse qu'il suscite, ou à la répugnance provoquée par les représentations qui nous blessent. C'est cette charge affective capable d'empêcher les représentations qui peut mener à la folie.

La théorie de la folie rend compte du même clivage dans le psychisme entre la force pulsionnelle et la représentation. Elle préfigure de façon saisissante, ici encore, nous dit Henry, les conceptions freudiennes. L'origine de la folie, c'est justement le refus de la volonté de laisser pénétrer dans la conscience une représentation qui lui est contraire en raison d'une répugnance ou d'un excès. Alors que l'expérience dite " normale » est conçue par Schopenhauer comme un processus douloureux et une impression pénible par lesquels (et bien qu'étant contraires à la volonté) des représentations - qui constituent ensemble et dans leur liaison nécessaire l'ordre du monde - doivent parvenir à la conscience et être reconnues comme telles, le refus de la volonté de laisser entrer dans l'esprit une représentation déplaisante et de la rejeter hors de la conscience provoquera un trou qui sera à l'origine de la folie. Il s'ensuit que, dans l'hypothèse d'une représentation ainsi refoulée, « le tissu des représentations qui forme le monde présente brusquement une lacune, un vide laissé par cette représentation absente $»^{57}$ et qu'il faudra combler par une autre qui n'est pas à sa place ou qui sera inventée à cette fin. Elle défera, en raison de ce déplacement, l'ensemble cohérent de représentations dans lequel consiste le monde. Cela sera le cas, qu'il s'agisse de celles qui définissent le présent ou de l'ensemble des représentations passées ou à venir. Ce qui est produit est une liaison arbitraire de phénomènes, un passé arrangé en cela que le contenu des souvenirs est falsifié et le fil associatif qui les lie est rompu, par exemple. Que ce soit en termes de mémoire ou d'anticipation, le naturant de la projection représentative est la volonté. Les représentations sont (ou ne sont pas) repoussées en raison de leur tonalité, en raison de ce qu'elles ont de désagréable ou d'intolérable, mais il faut aussi que le vouloir soit luimême originellement constitué en son être comme affectif, qu'il ne soit pas pur. "Ce n'est [...] jamais la volonté comme telle, un vouloir pur, qui écarte la représentation mais seulement un vouloir préalablement déterminé en soi comme affectif, comme répugnance, blessure, dégoût, honte $1{ }^{58}$ Cette doctrine, au moins dans la présentation qu'en fait Henry, ne fait-elle pas déjà signe vers une théorie de l'hallucination telle qu'elle est présente chez Freud ${ }^{59}$ ?

21 Si la doctrine de Schopenhauer anticipe la théorie freudienne du refoulement, elle en recèle aussi les apories. Henry met cela en évidence en constatant que pour permettre la venue à l'esprit d'une représentation ou pour l'interdire, il faut d'abord savoir ce qu'est 
une représentation et la connaître de quelque façon, ne serait-ce que pour apprécier sa convenance ou sa non convenance au désir. Mais, si elle ne connait rien et qui se définit par l'absence de connaissances (Erkenntnislosigkeit) comment la volonté pourrait-elle bien, sans représentation, posséder ou acquérir une telle connaissance nécessaire à rejeter une représentation? Cette aporie qui se trouve au coeur de la théorie du refoulement dès sa première formulation chez Schopenhauer atteint aussi toute conception qui voudra expliquer comment un pouvoir dépourvu de conscience peut bien écarter une représentation qu'il ne connaît d'aucune façon. C'est dire qu'elle se retrouve en toute philosophie faisant de l'inconscient le principe organisateur du psychisme et à plus forte raison, chez Freud et en psychanalyse. Mais elle ne subsiste que si nous restreignons le savoir à la conscience représentative. Si nous acceptons qu'il y a des formes de manifestation de ce savoir qui ne requièrent pas la représentation, l'aporie peut se résoudre. Henry nous indique cette solution en signalant que le savoir inhérent à la volonté, celui qui se trouve à l'oeuvre dans la formation ou la non formation des représentations, est affectif. ${ }^{60}$ C'est cette nouvelle théorie - proprement henryenne - qui rend intelligible le déplacement essentiel - entrevu par Schopenhauer mais non porté par lui à la clarté du concept - en vertu duquel toutes les facultés représentatives, l'oeil, la mémoire, la pensée, trouvent désormais leur principe dans un pouvoir qui n'est plus celui de la conscience intentionnelle : dans la vie. Or, celle-ci, est auto-affection. C'est là que se trouverait, paradoxalement le centre de l'inconscient: dans une auto-affection qui jamais ne devient inconsciente, mais qui n'est pas non plus à confondre avec la conscience, définie par la représentation. Il est possible de connaitre la représentation avant qu'elle ne soit parce que « ce n'est pas elle qui peut nous instruire sur elle-même et nous inviter à l'écarter, ce n'est pas son contenu représentatif, mais son affectivité $»{ }^{61}$ En sachant cela, et en suivant la réflexion de Michel Henry, nous avons désormais la possibilité de lire philosophiquement Freud : « en tant que l'acte proto-fondateur de l'extériorité qui sert de fondement à toute représentation possible s'auto-affecte, la formation de [cette extériorité] relève toujours de l'affectivité, l'affectivité de la représentation est celle du pouvoir qui la forme - ou qui ne la forme pas. Ce dernier cas est celui du refoulement dont l'énigme se dévoile à nos yeux $\$ .{ }^{62} \mathrm{Si}$ une représentation n'est pas formée, c'est seulement en fonction de son affectivité. Ce qui motive sa mise à l'écart de la conscience ce sont le déplaisir, la souffrance, voire la terreur liée à l'éventualité d'une représentation, non ce qu'elle représente. L'affect de la représentation était déjà là et c'est lui, et non pas la représentation qui a exclu le contenu représentatif, de lui faire subir le refoulement.

Ce n'est donc pas le contenu de la représentation qui va être connu avant qu'elle ne soit exclue. Ce n'est pas non plus une représentation qui écarte d'autres représentations. C'est une force étrangère à la représentation, comme l'affirme Schopenhauer. Mais, ajoute Henry, la force qui forme ou ne forme pas la représentation n'est pas soustraite à la phénoménalité, elle n'est pas réellement inconsciente. Elle est l' " Archi-phénoménalité " ${ }^{63}$ de l'Affect, caractérisée comme un " savoir sans représentation, savoir avant la représentation, savoir secret de toute représentation, qui sait déjà ce qu'elle va représenter, qui lui permet de le faire ou le lui interdit $»{ }^{64}$ Ce savoir sous-jacent, opérant, est celui de la vie en son essence phénoménologique propre. Il est l'affect consubstantiel à cette phénoménalité non ek-statique, celui qui ne saurait en être séparé en tant qu'elle est issue de la dynamique d'un sujet incarné. « En sorte que [...] placé au coeur de l'inconscient, l'affect détermine celui-ci comme phénoménologique en son essence et en son fond $» .65$ 


\section{Au-delà du principe de constance : pourquoi l'inconscient n'existe-t-il pas?}

23 Alors, comment comprendre en termes simplement affectifs le processus que Freud nomme refoulement? Si le poids de la vie qui s'auto-affecte se fait trop lourd, c'est-à-dire, s'il y a souffrance, la vie s'efforce d'échapper à soi, de se fuir. En raison du lien qui la lie à elle-même et qui ne peut être brisé, cela n'est pourtant pas possible et mène à un effort de changement. Remarquons que cette façon de faire de la pulsion un effort de modification inhérent aux tonalités affectives semble inverser le rapport entre la pulsion et l'affect tel que Freud en fait l'hypothèse depuis l'Entwurf. La lecture que Michel Henry fait de la façon dont Freud met en rapport la série plaisir-déplaisir, c'est-à-dire la dialectique fondamentale de l'affectivité, avec l'état de tension qui règne à la source pulsionnelle fait plutôt comprendre qu' " [e]n fin de compte, chez Freud lui-même, la phénoménologie fait s'écrouler le schéma spéculatif initial $»^{66}$ selon lequel l'affect est second par rapport aux quantités d'énergie dont il est un représentant. ${ }^{67}$ Pourquoi cela ? L'affectivité n'est plus seconde par rapport aux quantités d'énergie qui constituent ou supportent l'être de la pulsion, dès lors qu'elles " sont [...] la figuration des déterminations affectives fondamentales ${ }^{68}$ Ce qui permet à Michel Henry d'effectuer ce renversement apparent de la doctrine freudienne c'est Freud lui même, ou plutôt la théorie des pulsions telle qu'elle se dessine à la fois dans Le problème économique du masochisme et dans Au-delà du principe de plaisir. L'hypothèse affirmant que le plaisir est lié à une diminution d'excitation, le principe de constance, y est formellement contredite dès lors que Freud admet que le plaisir peut aussi correspondre à une augmentation de la tension et le déplaisir à sa diminution. Mais alors, si le principe de constance est mis à mal, quelle utilité peut-il y avoir à formuler l'hypothèse de la notion de pulsion dès lors que celle-ci est entendue comme ayant pour but de supprimer l'état de tension qui règne à la source de l'excitation endogène? Si nous voulons conserver au système freudien une quelconque cohérence, vaut-il mieux s'en débarrasser ou plutôt, comme le fait Michel Henry, interroge sa signification à partir de l'affectivité ? Dans ce deuxième cas, il semble qu'il faille reconnaître que l'affect fonde la pulsion. Que savons-nous, en effet, des quantités pulsionnelles qui déterminent les tonalités affectives ? Sont-elles vraiment exprimées par l'affectivité ? Il y a une difficulté méthodologique de la théorie psychanalytique qui subsiste chaque fois que Freud nous parle d'« excitation " pulsionnelle pour rendre compte de l'affect. Lorsque, à propos de la variation affective, nous parlons de l'excitation endogène, nous intégrons l'explication scientifique à l'expérience et nous semblons ainsi démontrer celle-là par celle-ci. Mais, phénoménologiquement parlant, éprouvons-nous une quantité d'excitation ou plutôt soit du plaisir soit du déplaisir ? Il n'y a pas de doute que c'est la tonalité affective qui constitue la réalité de notre vécu. «La quantité d'excitation n'est pas un au-delà du plaisir " ${ }^{69}$ c'est plutôt l'immédiateté de celui-ci ou de son contraire qui nous fait comprendre la signification de celle-là. Le terme " excitation " n'est qu'une façon de désigner le contenu phénoménologique de l'affect. C'est pour cette première raison que Henry nous dira que « le jeu des quantités et de leur variations, prétendument régi par le principe de constance, se ramène [...] en réalité à une régulation par la série plaisir-déplaisir, c'est-à-dire, à une dialectique de l'affectivité elle-même et se résorbe [...] entièrement en elle $»^{70}$ 
C'est donc, nous dit Henry, à la suite de Schopenhauer et de Nietzsche, qu'il faut situer ce qu'il y a de mieux dans les textes de Freud. C'est aussi dans ces textes que la situation clinique est présentée comme étant ancrée dans la dynamique affective du transfert, qui en est le principal moteur. Ils montrent que la représentation par l'analysé de sa propre situation, de ses conflits et de leur histoire, ne sert à rien aussi longtemps qu'une modification affective n'accompagne cette conscience, " c'est à dire [aussi longtemps] qu'une modification de la vie, n'est pas intervenue $» .^{71}$ L'acceptation intellectuelle du sens n'est rien sans une transformation affective qui ouvre de nouvelles possibilités de vie. Cette subordination s'étend à la détermination de la valeur de vérité des interprétations et des constructions, qui sera dépendante, elle aussi, de la teneur affective des rapports établis dans la communauté formée par l'analyste et l'analysant. Les meilleurs seraient justement ceux où le primat de l'affectivité affleure, où se fait jour « cette subordination de l'intellect à la vie ». La théorie et la thérapie psychanalytiques mettraient d'abord en évidence " cette subordination de la pensée représentative - perceptions, images, souvenirs, productions oniriques et symboliques, esthétiques et religieuses, etc. - et de tout ce qui se montre en elle, à un pouvoir d'un autre ordre $\gg .^{72}$

Compte tenu cette subordination de la pensée et de la représentation à l'affectivité, dégagée à partir de sa compréhension du refoulement, il est possible de mieux comprendre le statut que Michel Henry assigne à l'inconscient. Mais y a-t-il encore un sens quelconque à parler d'inconscient si nous savons que le principe qui y est à l'oeuvre est l'affectivité, et que celle-ci est toujours consciente, même dans les rêves? Quelle signification ce terme peut-il avoir après la critique de Michel Henry $?^{73}$ L'auteur de la Généalogie de la Psychanalyse, nous l'avons déjà remarqué, décèle sous la plume de Freud une double caractérisation du terme d'inconscient, selon le rapport que celui-ci entretient avec un savoir de la vie qui précèderait toute objectivité et qui serait celui d'une subjectivité absolue où toute représentation de trouve son origine. C'est ce savoir de la vie, qui est l'affectivité, en fonction duquel est inhibée ou formée une représentation, qui servirait de critère à la distinction de la double signification du concept d'inconscient. Il « voulait [...] dire deux choses totalement différentes selon qu'on se référait à l'obscurité en laquelle sombre inévitablement tout contenu conscientiel dès qu'il quitte le présent de l'intuition ou de l'évidence pour n'être plus qu'une représentation virtuelle, ou au contraire à la vie elle-même en tant qu'elle se dérobe par principe à la lumière de l'ek-stase $»{ }^{74}$ Selon Henry, cette double référence s'accomplit constamment chez Freud, chez lequel la simple latence des contenus représentatifs cède peu à peu la place à « une considération plus profonde qui thématise l'inconscient dans sa connexion à l'essence de la vie et au mode primitif de son accomplissement immanent : l'action, la force, la pulsion, l'Énergie $»{ }^{75} \mathrm{Si}$ la première caractérisation de l'inconscient succombe à la critique d'une phénoménologie radicale, l'autre, en revanche, se voit éclairée par celle-ci et se voit rangée parmi les formes que prennent l'immanence de la subjectivité vivante et son savoir immémorial et charnel. « Point n'est donc besoin pour rendre compte du refoulement de la mythologie des topiques ni de leurs personnages plus ou moins grossièrement empruntés au monde de la représentation, le savoir de la vie y suffit $» .^{76}$

26 Cette inutilité de la notion freudienne du refoulement s'étend aussi à l'inconscient de la représenteité entendu comme lieu des représentations refoulées qui gardent le caractère ek-statique En revanche, l'autre aspect de ce que Freud nomme inconscient a la plus grande importance, en tant qu'il rend compte de l'« en-deçà du monde où s'édifie 
intérieurement l'essence de tout pouvoir ${ }^{.77}$ " En-deçà du monde », cela veut dire, chez Henry, « dans l'invisible, dans l'immanence radicale de la subjectivité absolue ». C'est pourquoi, l'une des intuitions freudiennes les plus importantes, selon la réflexion de l'auteur de la Généalogie de la psychanalyse, est que la représentation est déterminée par le pathos de la vie, qu'elle détermine également le refoulement et le retour du refoulé. Cet " inconscient efficient $\aleph^{78}$ est la source irreprésentable de la représentation, le revers subjectif, inobjectivable, de l'objectité, l'épreuve de soi d'une vie s'exprimant par l'incessant des forces où se forme la teneur affective et sémantique des rapports entre sujets et de leur praxis. Là se trouve, également, la source de ce qui est donné comme monde à l'intentionnalité de la conscience, ainsi que celle des comportements humains. Cet inconscient, qui n'ek-siste donc pas, est - sous la plume de Henry - « le nom de la vie ".79

\section{NOTES}

1. Nous pouvons lire à ce propos l'ouvrage de Paul-Laurent Assoun, Freud, la philosophie, les philosophes, PUF, 1976.

2. Freud est un «héritier tardif» de la métaphysique occidentale, écrit Michel Henry dans sa Généalogie de Psychanalyse, Presses Universitaires de France, PUF, 1985, p. 5.

3. BORCH-JAKOBSEN, Mikel, «L'inconscient malgré tout ", in Les Études Philosophiques, 1, 1988, p. 1-36. Cet article, remanié, a été publié dans Le lien affectif, Paris, Aubier, 1992, et, récemment dans une troisième version comme chapitre de l'ouvrage Lectures de Michel Henry, enjeux et perspectives, LECLERC, Jean et JEAN, Gregori (Eds), Louvain-la-Neuve, Presses Universitaires de Louvain, 2014, p.123-158.

4. Voir à ce propos les passages que Léon Chertok et Isabelle Stengers y consacrent dans Le coeur et la raison: l'hypnose de Lavoisier à Lacan, Payot, 1989 et surtout l'article de François Roustang, " Une philosophie pour la psychanalyse ? ", in Critique, nº463, 1985, pp. 1172-1189 où nous trouvons l'affirmation de l'importance (op.cit. p.1172) presque décisive du travail de Michel Henry pour un renouveau de la clinique psychanalytique: «Par la force du raisonnement et par une impressionnante clarté, Michel Henry nous oblige à relire Freud pour accentuer la spécificité de sa découverte, lever les ambigüités où elle s'est parfois enlisée et déblayer le chemin de la thérapie devenue parfois impraticable. Une telle philosophie semble bien être aujourd'hui indispensable à la psychanalyse, si du moins cette dernière veut se penser avec rigueur et peutêtre se pratiquer correctement ».

5. BORCH-JAKOBSEN, Mikel, Le lien affectif, op.cit., p. 219.

6. HENRY, Michel, Phénoménologie Matérielle, Paris, PUF, 1990. Nous pouvons lire, à ce propos, le deuxième chapitre de la deuxième partie intitulé « Pour une phénoménologie de la communauté ».

7. HENRY, Michel, «La question du refoulement chez Schopenhauer ", Phénoménologie de la Vie. Tome II, op.cit., pp. 131-146 ; «Ricoeur et Freud : entre psychanalyse et phénoménologie» in Phénoménologie de la Vie. Tome II, Paris, PUF, 2003, pp. 163-183 ; «Signification du concept d'inconscient pour la connaissance de l'homme», in Auto-donation, Paris, Beauchesne, 2004, pp.87-110 ; "Phénoménologie et psychanalyse ", in Psychiatrie et existence, Pierre Fédida et Jacques Schotte (Éd.), Grenoble, Millon, [1991] 2007, pp. 95-107. 
8. HENRY, Michel, «Phénoménologie et psychanalyse ", dans Psychiatrie et existence, Pierre Fédida et Jacques Schotte (Éd.), Grenoble, Millon, (coll. Krisis), [1991] 2007, p. 95. Cet article est repris dans le tome V de Phénoménologie de la Vie, Paris, PUF, 2015.

9. « La vie n'est pas l'être. La vie n'est pas. Dans l'éprouver intérieur qui la constitue, elle naît et procede inlassablement en soi-même [...]. Et voici l'émergence, au sein de l'auto-affection, de l'Énergie primordiale » comme nous le rappelle Gabrielle Dufour-Kowalska dans son ouvrage intitulé Michel Henry, passion et magnificence de la vie, Paris, Beauchesne, 2003, p. 164.

10. HENRY, Michel, La Barbarie, Paris, Grasset, 1987, p. 163.

11. Ibid. Henry écrit aussi, dans l'introduction de sa Généalogie de la Psychanalyse [p.12], que « [1]a psychanalyse n'appartient donc pas au corps des sciences humaines auquel on la rattache aujourd'hui et dont elle sera ici soigneusement dissociée : elle en est bien plutôt l'antithèse ».

12. HENRY, Michel, La Barbarie, op. cit., p. 162.

13. Ibid.

14. HENRY, Michel, GP, p.8.

15. HENRY, Michel, GP, p. 5.

16. HENRY, Michel, Généalogie de la Psychanalyse, op.cit.,[désormais GP], p.8.

17. Ibid.

18. HENRY, Michel, GP, p. 384: « Résumons-nous: l'inconscient n'existe pas - si l'on écarte le fait, en l'occurrence la loi apriorique de toute phénoménalité extatique, que de la représentation presque tout le représenté se trouve exclu. Hors de la représentation ce représenté ne subsiste pas pour autant sous la forme de "représentations inconscientes », ces entités pour lesquelles le freudisme a imaginé des destins fantastiques ».

19. BORCH-JAKOBSEN, Mikel, «L'inconscient malgré tout », in Le lien affectif, op. cit., p. 211. « Les psychanalystes, pourtant, auraient tord de s'en tenir là. Et d'abord parce qu'il leur faudrait, alors, incriminer Freud lui-même. Car ce n'est pas Henry, c'est Freud, déjà, qui reconduisait l'inconscient dans la conscience. Que l'«inconscient» ne soit finalement rien d'autre que la conscience elle-même, c'est ce que Freud affirmait en effet très clairement en ouverture à l'essai sur «Le moi et le ça», dans une proximité fort troublante aux thèses de Michel Henry. L'opposition du moi conscient et de l'inconscient refoulé, y reconnaissait-il, ne saurait nous fournir le dernier mot de la névrose (ni du même coup de la psyché en général), puisque la «résistance» que le premier oppose au second est elle-même inconsciente: le névrosé n'a aucune conscience de son propre refoulement... Et il faut donc admettre [...] que le moi est lui-même inconscient, inconscient de lui-même. [...] Ce qui, il est vrai ne signifiait nullement que l'inconscient dût désormais être résorbé dans la conscience [...]».

20. Ibid.

21. FREUD, Sigmund, "Contribution à l'histoire du mouvement psychanalytique ", in Essais de Psychanalyse, Paris, Payot, 1936, p. 273.

22. FREUD, Sigmund, « Pulsion et destins des pulsions », dans Métapsychologie, p. 18-19.

23. Je reprends ici la remarque de François Roustang telle qu'elle est formulée dans l'article consacré à Henry cité plus haut et selon laquelle « à [sa] connaissance personne n'avait jusqu'à présent proposé une ontologie originale capable à la fois de situer l'oeuvre de Freud dans le courant philosophique auquel elle se rattache explicitement [...] et de mettre par là même en pleine lumière l'originalité de cette oeuvre en tant qu'elle est susceptible de fonder une pratique ».

24. FREUD, Sigmund, « Pulsion et destins des pulsions », in Métapsychologie, p. 14.

25. Ibid.

26. Ibid.

27. FREUD, Sigmund, "Le refoulement ", in Métapsychologie, trad. de l'allemand par Jean Laplanche et J.-B. Pontalis, Gallimard, (coll. « Folio »), p.47.

28. HENRY, Michel, GP, p. 370. Fr. Roustang insiste longuement sur cet aspect de la théorie freudienne en commentant l'essentiel de l'ouvrage de Henry («Une philosophie pour la 
psychanalyse ?», op. cit., p. 1181) : «Freud distingue [...] très nettement les manières de parler et le langage correct. S'il est habituel de dire [...] que les affects sont inconscients, cette formule est tout simplement fausse. Ce qu'il faut dire, selon Freud, et qui est tout différent, c'est qu'il arrive qu'une «motion d'affect ou de sentiment soit perçue, mais méconnue.» Il est de plus à remarquer qu'il n'est pas seulement ici parlé d'affects, mais de sentiments et de sensations. C'est donc toute la vie affective qui est convoquée et qui, au sens strict, ne saurait être caractérisée comme inconsciente ».

29. HENRY, Michel, GP, p. 370.

30. HENRY, Michel, GP, p. 373.

31. C'est ce qu'avance Mikel Borch-Jakobsen, alors même qu'il souligne l'importance de la thèse de Henry pour une psychanalyse « devenue exsangue à force de s'être confiée aux seuls pouvoirs de la représentation, du langage, du signifiant» : Henry [...] dissout et pour tout dire «relève» l'énigmatique altérité de l'inconscient en pure et simple et vivante conscience de soi. Une chose est en effet de dire que l'inconscient est l'irreprésentabilité de la conscience comme telle, est l'irrépressible passivité de son affect. Autre chose d'affirmer que cette passion est bonnement l'envers de l'auto-affection de la vie par elle-même - bref, l'«effet» d'une activité ou d'une spontanéité plus enfouie (plus subjectale, donc) que celles de la volonté ou du désir «au sens de la représentation»». BORCH JAKOBSEN, Mikel, Le lien affectif, op. cit. p. 221.

32. HENRY, Michel, «Psychanalyse et phénoménologie », dans Psychiatrie et existence, op. cit., p. 102.

33. HENRY, Michel, GP, 10.

34. HENRY, Michel, « La question du refoulement chez Schopenhauer », dans PV-II, p. 131.

35. HENRY, Michel, « La question du refoulement chez Schopenhauer », dans PV- II, p. 133.

36. HENRY, Michel, GP, p. 225.

37. HENRY, Michel, « La question du refoulement chez Schopenhauer », dans PV-II, p. 131.

38. Voir, plus haut, la note 28.

39. Ibid., p. 134.

40. Ibid.

41. Ibid.

42. Ibid.

43. Ibid.

44. Ibid.

45. Ibid.

46. Ibid.

47. "La question du refoulement chez Schopenhauer ", dans PV-II, p. 135.

48. HENRY, Michel, « La question du refoulement chez Schopenhauer », dans PV-II, p. 136.

49. HENRY, Michel, GP, p. 226.

50. Ibid.

51. HENRY, Michel, « La question du refoulement chez Schopenhauer », dans PV-II, p. 137.

52. Ibid.

53. Ibid.

54. HENRY, Michel, GP, p. 231.

55. Ibid.

56. HENRY, Michel, « La question du refoulement chez Schopenhauer », dans PV- II, p. 136.

57. Ibid., p. 136.

58. HENRY, Michel GP, p. 233.

59. Pensons ici au rapport entre résistance, transfert et répétition. Sans hallucination, comment l'actualité vécue pourrait-elle être investie par ce qui ne parvient pas à la forme représentative? L'hallucination serait le résultat d'une perception travaillée du dedans et cela ne semble être possible que grâce à un l'affect capable de (dé)former le champ perception-langage. L'affect du transfert est un Agieren de la pulsion, un «agir» du refoulé, et ceci veut dire avant toute chose 
qu'il court-circuite la représentation, nous dit Freud. Le fait oublié ne reparait pas sous forme de souvenir, mais sous forme d'Agieren. C'est cette formation du champ perception langage qui rend possible l'investissement du refoulé sous forme d'une action remplaçant le souvenir. Henry traduit cette Agieren en termes de force inhérente à l'auto-affection et en fait l'invisible où prend forme l'extériorité (visible) du monde. Freud ne dit-il pas que le malade, un peu comme dans un rêve, accorde aux expériences d'éveil de ses motions inconscientes actualité et réalité, qu'il veut agir ses passions sans égards pour la situation réelle?

60. Mikel Borch-Jakobsen, dans «L'inconscient malgré tout» (in Le lien affectif, op. cit., p. 217) attire notre attention sur des aspects de la pensée freudienne qui nous aident à comprendre la lecture qu'en fait Henry en passant par la réflexion de Schopenhauer : «[P]eut-on encore dire que le patient, éprouvant tel ou tel affect, ne veut rien savoir des représentations qui le hantent ? Ne faut-il pas plutôt admettre qu'il ne peut rien en savoir - du moins savoir au sens de la représentation? Et n'est-ce pas là la clef de sa singulière « résistance » à l'analyse ? Aussi est-ce ailleurs, dans l'«agir» involontaire et irreprésentable de l'affect, qu'il faut chercher la solution. Il se trouve en effet que l'affect, comme Freud le dit ailleurs du «travail du rêve», ne pense pas. Il est agi, selon une efficience qui n'a justement rien à voir avec une volonté délibérant avant de se mettre en mouvement et qui ainsi saurait ce qu'elle veut (définition traditionnelle de la voluntas comme appetitus intellectualis)».

61. HENRY, Michel, « La question du refoulement », dans PV-II, p. 144.

62. HENRY, Michel, GP, p. 233-234.

63. Ibid.

64. HENRY, Michel, GP, p. 234.

65. HENRY, Michel, GP, p. 10. On aurait pourtant tord, nous dit M. Borch-Jakobsen (Le lien affectif, op.cit., p.220), « (les psychanalystes auraient tord) de reprocher à Michel Henry de se limiter à une analyse de la conscience. La sûreté quasi somnambulique avec laquelle il rejoint certaines des propositions les plus problématiques du dernier Freud l'atteste suffisamment : cette inconscience aveugle de l'affect n'est autre que l'inconscient, dans son énigme ainsi ravivée ».

66. HENRY, Michel, GP, 377.

67. Lisons à ce propos, l'entrée « Affect » du Vocabulaire de Psychanalyse, où nous apprenons que «l'affect est l'expression qualitative de la quantité d'énergie pulsionnelle et de ses variations». LAPLANCHE et PONTALIS, Vocabulaire de Psychanalyse, Paris, PUF, 1971, p. 12.

68. HENRY, Michel, GP, 375.

69. Ibid.

70. HENRY, Michel, GP, p. 376. « Autre façon de dire, une fois de plus, remarque M. BorchJakobsen (Le lien affectif, op.cit. p. 218) que l'affect (l'agir, la répétition, le transfert) ne connaît ni délai, ni temps, «ni négation, ni doute, ni degré dans la certitude». Toutes ces «propriétés particulières de l'inconscient» selon Freud conviennent éminemment à l'affect, qui est ainsi comme l'épreuve même de l'inconscient - et son épreuve intégralement consciente... L'affect, doit-on dire, est le singulier Cogito de l'inconscient, l'affect est la singulière inconscience du Cogito (de fait, a-t-on assez remarqué que les «propriétés» de l'inconscient sont celles-là mêmes du Cogito, à sa pointe «extravagante» : instantanéité, immédiateté, positivité, indubitabilité ?) » .

71. HENRY, Michel, GP, p. 385.

72. HENRY, Michel, GP, p. 10.

73. C'est là " le rasoir d'Henry », comme le dit Mikel Borch-Jakobsen (Le lien affectif, op. cit., 214) reprenant la remarque de Fr. Roustang, qui affirme que " nous pourrons désormais relire l'oeuvre de Freud, ayant à notre disposition un authentique rasoir d'Occam, pour mettre, d'une part, ce qui est intuition géniale ou transposition imaginée de la chose qui nous concerne et dont il est si difficile de parler, et d'autre part, ce qui est retour à l'infatigable effort de la philosophie occidentale pour tout ramener dans les tenailles de la représentation». («Une philosophie pour la psychanalyse ?», op. cit., p. 1189). 
74. HENRY, Michel, GP, p. 388.

75. HENRY, Michel, GP, p. 388.

76. HENRY, Michel, GP, p. 234.

77. HENRY, Michel, GP, p. 392.

78. HENRY, Michel, GP, p. 392.

79. HENRY, Michel, GP, p. 348. Fr. Roustang souligne ainsi, («Une philosophie pour la psychanalyse?», op.cit., p.1181) en commentant la thèse de Michel Henry selon laquelle l'inconscient n'ek-siste pas qu' « il est clair que l'attribut inconscient s'applique soit à la pulsion, soit à la représentation. Mais si, d'une part, comme il a été montré, la pulsion est une hypothèse non nécessaire pour rendre compte de l'affectivité et si, d'autre part, ainsi qu'il a été dit plus haut, la représentation n'est inconsciente que par le fait qu'elle peut n'être pas actuellement présente à la conscience - fait qui n'a pas préoccupé Freud outre mesure et qu'il a désigné du terme de préconscient - il faut conclure que l'inconscience n'existe pas ou, ce qui est moins abrupt, mais pas moins sévère, que l'on ne peut parler d'inconscient que par négligence de langage ». Ajoutons à cela le commentaire de M. Borch-Jakobsen («Le lien affectif», op.cit., pp. 220-221) qui nous fait comprendre non seulement pourquoi, en un sens, selon Henry, Freud est tributaire d'une certaine lecture du cogito cartésien, mais aussi pourquoi, en pensant la subjectivité à partir de l'auto-affection de la vie, la phénoménologie de Henry passe outre la distinction entre conscient et inconscient, pouvant affirmer que le second n'existe pas. " L'inconscient ne s'oppose pas à la conscience, pas plus que la conscience ne s'oppose à l'inconscient, ils sont l'un comme l'autre, en-deçà de cette opposition, qui est l'opposition représentative comme telle. Et ils y résistent tenacement, obstinément. Ce que Freud, sous les titres du transfert et de la répétition, s'acharnait à penser comme une résistance à cette obposition là, à la prise de conscience comme saisie représentative. C'était, comme le dit bien Henry, l'affect, le profond oubli de l'affect, dans son insurmontable opposition à la représentation, à la remémoration, à la «temporalité ek-statique» en général ».

\section{RÉSUMÉS}

The phenomenological reading of psychoanalysis undertook by Michel Henry is surprising in several ways. By inscribing Freud in the philosophical lineage and by affirming that his theses on the unconscious take over (resume), ignoring it, two opposite movements of western metaphysics, Henry intends to show that psychoanalysis has to overcome the primacy of representation in order to get rid of the contradictions that traverse it and that make it a prisoner of a philosophy of consciousness. Without fully rejecting the Freudian theory, the author of the Genealogy of Psychoanalysis reformulates the meaning of the meta-psychological notions according to his theses on the cogito, subjectivity and the auto-affection of the living. In this article we retraced the stakes of this redefinition of drives, affect and the unconscious considering his reflexions on repression.

\section{INDEX}

Mots-clés : vie, affect, pulsion, inconscient, Michel Henry

Keywords : life, affect, drive, unconscious, Michel Henry 


\section{AUTEUR}

\section{NUNO MIGUEL PROENÇA}

CHAM, FCSH, Universidade NOVA de Lisboa, Universidade dos Açores, Portugal. Bolseiro de PósDoutoramento da FCT - SFRH/BPD/110895/2015.

nunomiguelproenca@gmail.com

Post-doctorant au CHAM, FCSH, Universidade NOVA de Lisboa, Universidade do Açores (FCT SFRH/BPD/110895/2015) et docteur en philosophie de l'EHESS - où il a étudié avec Fernando Gil. Il a enseigné au Collège International de Philosophie (2006-2008) et a été membre du Centro Português de Psicanálise (2010-2014). Depuis 2014, il enseigne la philosophie à l'APPSI. À ce jour, il a publié Qu'est-ce que l'objectivation en psychanalyse? (L'Harmattan, Paris, 2008), Wittgenstein, a prova e a actividade matemática: uma introdução (Centro de Filosofia das Ciências da Universidade de Lisboa, 2009), articles et chapitres de livres, quelques traductions scientifiques et un roman. Postdoctoral researcher at the CHAM, FCSH, Universidade NOVA de Lisboa, Universidade dos Açores (FCT SFRH/BPD/110895/2015) and a PhD in Philosophy from the EHESS - where he studied with Fernando Gil. He taught at the Collège International de Philosophie (2006-2008) and was a member of the Centro Português de Psicanálise (2010-2014). Since 2014, he teaches philosophy at the APPSI - the Portuguese branch of the International Association for Relational Psychotherapy and Psychoanalysis. To this day, he has published Qu'est-ce que l'objectivation en psychanalyse? (L'Harmattan, Paris, 2008), Wittgenstein, a prova e a actividade matemática: uma introdução (Centro de Filosofia das Ciências da Universidade de Lisboa, 2009), articles and book chapters, some scientific translations and a novel. 